\title{
Embedding neuroscience in psychiatry training
}

\author{
Christopher 0’Loughlin (i)
}

\begin{abstract}
SUMMARY
Training in neuroscience is vital to the future of psychiatry as a medical specialty. Trainees and trainers alike demonstrate a desire to keep up to date with developments in the associated scientific fields. Neuroscience increasingly underpins clinical assessments, treatment options and patients' expectations. Psychiatry training in the UK can embrace neuroscience at many levels, from discussing patient presentations with supervisors, to teaching programmes supported by the Royal College of Psychiatrists' activities. Although challenges remain, neuroscience literacy enhances the specialty and will improve patient care.
\end{abstract}

\section{KEYWORDS}

Education and training; cognitive neuroscience; novel CNS drugs.

Neuroscience, the study of the biological mechanisms underlying brain function, is coming of age in psychiatry training. Neuroscience encompasses a wide range of techniques and disciplines, including structural and functional neuroimaging, genetics and epigenetics, intracellular biology, neuroimmunology, endocrinology, pharmacology, cognitive and behavioural psychology, and evolutionary theory. Neuroscience uses the latest technological possibilities, from brain organoids to computational modelling. The accelerating advance in our understanding of brain function over the past 20 years has been incredible and, to clinicians, possibly overwhelming. In the UK, the Royal College of Psychiatrists' (RCPsych's) Neuroscience Project, supported by The Gatsby Foundation and The Wellcome Trust, has been leading change in the engagement of clinicians with neuroscience since 2016.

\section{Psychiatry and neuroscience are intrinsically linked}

Psychiatry, the branch of medicine that treats people with mental disorders, is intrinsically linked to developments in neuroscience. Identifying the neurobiological underpinning of symptoms and the mechanisms by which physical, psychological and social adversity translate into recognisable clinical presentations, sometimes years or decades later, is fundamental to the progress of our specialty. This applies both in understanding the difficulties our patients have and in providing appropriate, and novel, interventions. Clinical presentations may appear to arise from complex and heterogeneous brain diseases, such as schizophrenia, or the normal functioning of compensatory self-regulating mechanisms leading to symptom production and functional impairment.

\section{But what has neuroscience ever done for psychiatry?}

However, despite the optimism and successes in the field it is still possible to ask as psychiatrists in 2020, 'What has neuroscience ever done for us?' (Kingdon 2020). To answer this, a rehearsal of some of the direct areas of relevance of neuroscience to clinical practice is worthwhile. Typically, those doubting that this is a fertile area of inquiry will point to the lack of a definitive clinical test that can confirm common diagnoses in psychiatry, or claim that, despite all the discoveries, no new treatments have emerged. Such a stance neglects significant clinical domains where diagnosis and treatment are clearly informed by neuroscientific developments: psychiatrists who work with people with intellectual disabilities, with new-onset psychosis or with dementia routinely rely on imaging, electroencephalogram (EEG) studies or immunological testing to guide management strategies. Likewise, the development of aripiprazole as a widely used first-line treatment for psychosis hinged on the requirements of finding a medication that reduces dopamine $\mathrm{D}_{2}$ receptor function without the wider side-effects of previous treatments (Casey 2017).

\section{A new generation of trainees - and patients}

When talking with psychiatry trainees, it appears that the groundswell of interest in neuroscience is driven by the knowledge that a full understanding of patients' presentations is enhanced by completing the biopsychosocial formulation, with a solid basis in biological functions and structures. This holds true for the brain abnormalities seen in dementia and schizophrenia, the effects of childhood trauma
CLINICAL REFLECTION
Christopher O'Loughlin is a consultant psychiatrist with Cambridgeshire and Peterborough NHS Foundation Trust, Cambridge, and Head of the School of Psychiatry for the East of England region of Health Education England, UK. Correspondence Dr Christopher O'Loughlin. Email: christopher. oloughlin@cpft.nhs.uk

First received 27 Mar 2020 Accepted 9 Apr 2020

Copyright and usage The Authors 2020 
on development and, increasingly, the understanding of specific neural circuitry driving symptom profiles in, for example, obsessive-compulsive disorder. Though important, the interest does not solely derive from a utilitarian demand for tests and drugs.

Clinicians will certainly be regularly challenged by patients who bring their own understanding of their illness to the clinic. This will often be couched in neurobiological terms, given the wide reach of neuroscience in the media, and psychiatrists need to be able to discuss the developments in the field in a supportive and informed manner. Patients can reasonably expect doctors to understand their condition and be able to explain it. Psychiatry trainees looking to the future believe that the improvements in understanding the biological processes behind our patients' difficulties enhances the specialty, makes it easier to campaign for real parity of access to services and has the potential to reduce the stigma that our patients face on a daily basis. This will only come about if psychiatrists have the knowledge and skills to shape the wider landscape in political health discussions.

\section{Advances in neuroscience training}

How can we support new psychiatrists to learn neuroscience in their training? The changing curriculum being developed by the Royal College of Psychiatrists and the updating of the examinations for membership will undoubtedly drive trainees' learning objectives. Trainees can access a range of online resources, particularly those on the National Neuroscience Curriculum Initiative (NNCI) in the USA and on Trainees Online (TrON), the RCPsych's learning resource, which is freely available to registered trainees.

Of course, the purpose of neuroscience training for psychiatrists is not to create a sterile and separate body of knowledge learnt from books or papers but to engage with conversations in the clinic. Trainees will find a far greater resonance in their learning when they are engaged with aspects of neuroscience on a day-to-day basis. This can be achieved in their clinical experience, their supervision with trainers and in discussions about how our current knowledge, despite limits, leads to improved patient care.

Clinician-trainers need themselves to be empowered to start discussions with trainees about the neurobiological processes behind patient presentations and how they inform our understanding and treatment options. To this end in the UK the RCPsych's Neuroscience Project has been supporting a series of 'Brain Camps' across the four nations that have reached over 200 trainers and provide an immersive 1-day experience combining the latest clinical neuroscience research with innovative educational strategies. The ongoing support for both trainees and trainers is then consolidated through regional trainee neuroscience champions, the development of regional networks linking brain researchers with trainers, a national conference and the trainee-led newsletter PSynapse (www. rcpsych.ac.uk/training/neuroscience-in-training/ neuroscience-resources).

The aspiration is that trainees and trainers will have discussions about the specific relevant aspects of the biological nature of each patient's presentation as routine and, in combining this with the psychological and social processes that are already discussed, be able to communicate effectively with patients about their conditions and use this knowledge to make treatments more appropriate and effective.

\section{The future: challenges and opportunities}

Of course, there will be challenges to overcome as neuroscience is increasingly integrated into clinical care. Knowledge gaps in the field remain considerable and will be increasingly called out. Progress requires a new generation of informed clinicianscientists to focus basic science research questions in clinical areas where there is great need for improved understanding and treatments. We will need to encourage some of those from the new generation of trainees versed in neuroscience to work in this vital area. Mental health services should also be rightly demanding resources for investigations such as imaging, biomarkers and personalised pharmacological medicine, which are accepted as standard in other medical specialties.

Neuroscience has the potential to make psychiatry more than it has been: to improve our understanding of symptoms, conditions and patient outcomes, to integrate physical and mental healthcare, and to provide mental health services with further strength and justification for the resources it, and our patients, deserve.

\section{Declaration of interest}

None.

An ICMJE form is in the supplementary material, available online at https://doi.org/10.1192/bja. 2020.27 .

\section{References}

Casey AB, Canal CE (2017) Classics in chemical neuroscience: aripiprazole. ACS Chemical Neuroscience, 8: 1135-46.

Kingdon D (2020) Why hasn't neuroscience delivered for psychiatry? BJPsych Bulletin [Epub ahead of print] 13 Feb. Available from: https:// doi.org/10.1192/bjb.2019.87. 